\section{Here be no dragons}

\section{David Ruelle}

Nature presents us with a bewildering variety of complex systems, from the Earth's atmosphere, to the stock market, to the anatomy of the fly. Their complexity appears in different ways and for different reasons. One cannot hope for a general theory to explain all complex systems, but theoretical ideas may provide insight into some. The idea of biological evolution helps us understand the complexity of living beings; the idea of chaos helps make sense of some complicated, irregular, time evolutions such as the flow of a turbulent fluid.

How can you obtain oscillations with a complicated, irregular, time dependence? One way (but not the most interesting) is to put together many oscillators with different frequencies. Another is to use just a few oscillators (say three), and throw in a nonlinear interaction, resulting in a deterministic time evolution with sensitive dependence on initial condition. What is that? Well, in a deterministic time evolution, if you know the state $x(0)$ of the system at time 0 , you can predict its state $x(t)$ at time $t$ with complete precision. But if there is an imprecision $\epsilon(0)$ in the initial condition $x(0)$, the imprecision $\epsilon(\mathrm{t})$ in $x(t)$ may grow exponentially with $t$, at least for a while. This is sensitive dependence on initial condition - in the case of a pencil one is attempting to balance on its tip, the initial condition determines the side to which it falls. The surprise is that in some systems there is sensitive dependence on initial condition whatever the initial condition. This is called chaos: a chaotic system is unstable everywhere. Obviously, the evolution in time of a chaotic system has limited predictability, and the system undergoes

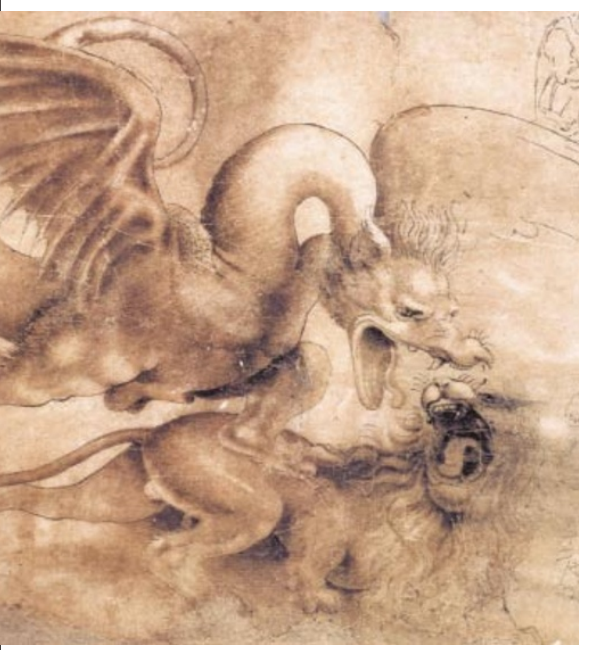

A vision from hypothetical history? irregular oscillations (if they were regular they would be predictable).

The mathematical possibility of chaos was understood 100 years ago by Hadamard and Poincaré, but theory and applications were developed more recently. Apart from a remarkable paper by Lorenz in 1963, the flow of papers started in the 1970s. Computer studies then made it clear that chaos is rather a common phenomenon. We now know that hydrodynamic turbulence is chaotic, which certainly puts the subject in a new perspective. We also know that the motion of the Earth's atmosphere has sensitive dependence on initial condition, which sets fundamental limits on weather predictions. (Although we have good knowledge of short-term weather evolution, understanding longer-term climate evolution largely escapes us.) Among the more recent successful applications of chaos theory, the most spectacular relate to the Solar System: the parameters of the orbit of the Earth vary chaotically with a characteristic time of about five million years ( short on geological timescales), which has implications for palaeoclimates.

The basic concepts and methods of chaos have become widely accessible, being successfully applied to various interesting, relatively simple, precisely controlled systems. Other attempts, for example applications to the stock market, have not yielded convincing results: here our understanding of the dynamics remains inadequate for a quantitative application of the ideas of chaos.

There are philosophically important consequences of chaos. Poincaré noted that the uncertainty of our predictions justifies a probabilistic description of the world. But we can now go further: we can compute the probabilities of some historical events, such as the chance of rain on Piccadilly Circus or Times Square, given what is known of the state of the Earth's atmosphere 48 hours earlier.

This leads me to digress on the tantalizing topic of historical probabilities. Philosophers and scientists have speculated about the probability of life arising on Earth or elsewhere. Can one construct a probabilistic history of the world - the formation of the Solar System, life on Earth, or human history? In principle one can speak about the conditional probability of a certain event, given what is known about the state of the Universe at some earlier time. Two things are needed for such a probability to be of interest: first, that it can be estimated at all; and second, that the estimate doesn't depend too much on insignificant details of the assumed earlier state of the Universe. The estimate can involve not just chaos theory, but other ideas of smooth dynamics, quantum uncertainty,
Chaos

"The idea of biological evolution

helps us understand the complexity

of living beings; that of chaos helps

make sense of irregular time

evolutions such as those of a

turbulent fluid."

and further concepts yet to be developed. In this respect it is encouraging that some experts can make useful guesses about the probable evolution of the stock market, or the outcome of a political election.

To take a concrete example, instead of asking when biological evolution decided that terrestrial vertebrates have four limbs, we might ask what the probability was that at some prescribed earlier time, say the end of the Cambrian, they would develop six limbs. More precisely, can one hope for a reasonable description of life at the end of the Cambrian, such that the probability of the number of limbs of future terrestrial vertebrates could be estimated in a stable manner? Animals with six limbs would be interesting because they could adapt two limbs for manipulative purposes (centaurs) or for flying (dragons). But as centaurs and dragons do not exist, it is tempting to say that they must have had negligible probability of coming into being, and dismiss the question. One cannot so easily dismiss the problem of how life on Earth would have evolved if the great cataclysm and extinction at the end of the Cretaceous had not taken place. Are such problems outside the scope of scientific investigation? At the very least, their solution would require far greater understanding of biological evolution.

Questions of hypothetical history, or historical probabilities, are often unanswerable, at least at present. Yet these questions are not meaningless. We might one day make sense of some probabilities that arise in the history of life on Earth or in other areas. After all, such probabilities can already be estimated in the simple cases of weather prediction and astronomy of the Solar System.

David Ruelle is honorary professor at the Institut des Hautes Etudes Scientifiques,

91440 Bures sur Yvette, France.

\section{FURTHER READING}

Ruelle, D. Chance and Chaos (Princeton Univ. Press, Princeton, 1991; also in Penguin, 1993). Cvitanovic, P. Universality in Chaos (Adam Hilger, Bristol, 1984). Hao, B.-L. Chaos II(World Scientific, Singapore, 1990). Laskar, J. Large scale chaos and marginal stability in the solar system. In Proc. XIth ICMP Meeting, Paris, July 1994, 75-120 (International Press, Boston, 1995); Celestial Mechanics64,115-162 (1995). 